\title{
Acute Intestinal Necrosis due to Diaphragmatic Hernia after Radiofrequency Ablation for Hepatocellular Carcinoma: A Case Report and Literature Review
}

\section{Yaoqing Li}

Shaoxing People's Hospital https://orcid.org/0000-0001-8562-9251

\section{Liyijing Shen}

Shaoxing People's Hospital, Shaoxing Hospital of Zhejiang University

Kelong Tao

Shaoxing People's Hospital, Shaoxing Hospital of Zhejiang University

\section{Shanlu Yu}

Shaoxing People's Hospital, Shaoxing Hospital of Zhejiang University

Guangen Xu ( $\nabla$ xuguangen1972@163.com)

Shaoxing People's Hospital https://orcid.org/0000-0003-2056-4308

\section{Case report}

Keywords: Radiofrequency Ablation, Diaphragmatic Hernia, Hepatocellular Carcinoma, Intestinal Necrosis

Posted Date: March 16th, 2021

DOI: https://doi.org/10.21203/rs.3.rs-307656/v1

License: (c) (i) This work is licensed under a Creative Commons Attribution 4.0 International License.

Read Full License 


\section{Abstract}

Background: Radiofrequency ablation is widely used as an effective and minimally invasive treatment of hepatocellular carcinoma. The complications of radiofrequency ablation are mainly associated with needle damage or local thermal damage, while the reports of long-term complications of radiofrequency ablation are relatively rare.

Case Report: This report presents a case of hepatocellular carcinoma with diaphragmatic hernia after radiofrequency ablation. A 60-year-old male came to the hospital with sudden abdominal pain for one day, who had received radiofrequency ablation and transcatheter arterial chemoembolization treatment for hepatocellular carcinoma located in segment 8 as an initial treatment 52 months ago. Computed tomography showed the right diaphragmatic hernia, small intestine intruding into the chest with intestinal obstruction. The patient underwent an emergency diaphragmatic repair with bowel resection and was successfully discharged 1 week after the operation.

Conclusion: Diaphragmatic hernia is a long-term complication of radiofrequency ablation. Surgical treatment is recommended as the first choice when conditions permitted.

\section{Background}

Hepatocellular carcinoma (HCC) is one of the most common malignant tumors in the world ${ }^{[1]}$. It mainly depends on local treatment, including surgical resection, radiofrequency ablation (RFA) and transcatheter arterial chemoembolization (TACE) ${ }^{[2,3]}$. Recent studies have shown that RFA, as a minimally invasive treatment, has been widely used in the local treatment of $\mathrm{HCC}$, and the indications have been gradually broadened $^{[4,5]}$. Long-term follow-up data ${ }^{[6]}$ have shown that the therapeutic effect of RFA is comparable to surgical resection, while the complications are far less than surgical resection. The major complications of RFA including bleeding, liver abscess, bowel perforation, pneumothorax, and tumor implantation metastasis ${ }^{[7]}$, etc. Most of these are short-term complications, while the long-term complications are relatively rare $^{[8]}$.

Diaphragmatic hernia (DH), as a long-term complication of RFA, is mostly caused by diaphragm injury at the puncture site induced by local high temperature ${ }^{[7]}$. While acquired $\mathrm{DH}$ present as the abdominal organs projecting into the chest through a pleural defect, as a result, patients come to the hospital because of abdominal pain or chest tightness. However, the relevant reports are few, while the treatment plan and prognosis are also different.

A case of acute intestinal obstruction with necrosis caused by DH after RFA was reported. The patient underwent emergency surgery to remove the necrotic intestine and repair the diaphragmatic defect and recovered after surgery. We also reviewed the reports of 12 cases of DH after RFA and compared the differences in clinical features and prognosis. 


\section{Case Report}

A 60-year-old male came to the hospital with sudden abdominal pain for one day. He had a history of hepatitis B virus-related liver cirrhosis for more than 20 years and long-term oral lamivudine tablets (100mg, qd, p.o.). He had received RFA and TACE treatment for HCC located in segment 8 as an initial treatment for HCC 52 months before admission, postoperative long-term oral sorafenib tablets (400mg, bid, p.o.), and regular outpatient examinations (abdominal computed tomography (CT) every 3 months, alpha fetoprotein (AFP) monthly) showed no tumor recurrence or diaphragmatic hernia.

After the patient entered the emergency room, abdominal CT was completed immediately, showed that right diaphragmatic hernia, small intestine intruding into the chest, intestinal obstruction, little effusion in the right thoracic and abdominal cavity, cirrhosis, and postoperative changes of RFA and TACE (Fig. 1). Laboratory examination: alanine aminotransferase (ALT) : $20.5 \mathrm{U} / \mathrm{L}$, aspartic aminotransferase (AST) : $29.2 \mathrm{U} / \mathrm{L}$, alkaline phosphatase (ALP) : $70.7 \mathrm{U} / \mathrm{L}$, gamma pancreatic acyl transferase (GGT) : $56.4 \mathrm{U} / \mathrm{L}$, total bilirubin (TBIL) : $12.4 \mu \mathrm{mol} / \mathrm{L}$, direct bilirubin (DBIL) : $6.5 \mu \mathrm{mol} / \mathrm{L}$ and indirect bilirubin (IBIL) $: 5.9$ $\mu \mathrm{mol} / \mathrm{L}$, total protein (TP) : $81.4 \mathrm{~g} / \mathrm{L}$, albumin (ALB) :43.2 g/L. Prothrombin time (PT) : $15.2 \mathrm{~s}, \mathrm{D}$-dimer (DD) : $1.74 \mathrm{mg} / \mathrm{L}$. Hepatitis B virus surface antigen (HBsAg) : positive, hepatitis B virus e antibody (HBeAb) : positive, hepatitis B virus core antibody (HBcAb) : positive. Platelet count (PLT) : $97 * 10^{9} / \mathrm{L}$, white blood cells and hemoglobin were maintained at normal levels, AFP was normal. The patient's Child-Pugh score was 6 (grade $A)$ at that time.

The patient underwent exploratory laparotomy with general anesthesia in an emergency. During the operation, it was observed that there was a local weak defect about $3 \mathrm{~cm}$ long in the right diaphragmatic muscle. The small intestinal hernia entered the right thoracic cavity $20 \mathrm{~cm}$ away from the ileocecal valve and was locally incarcerated. The incarcerated intestine was necrotic with dark color, no peristalsis, and the proximal intestinal was dilated. There was about $200 \mathrm{ml}$ bloody effusion in the right thoracic cavity and $50 \mathrm{ml}$ pale yellow effusion in the abdominal cavity (Fig. 2). Enterectomy combined with diaphragmatic repair was performed, and the operation was successful. Thoracic and abdominal drainage tubes were placed during the operation, and patient was successfully discharged 1 week after the operation.

\section{Discussion}

According to the National Comprehensive Cancer Network (NCCN) guidelines, RFA is recommended for patients with HCC whose liver function or systemic conditions are poor and are not suitable for surgical resection, with an expected survival comparable to the surgery and fewer complications ${ }^{[9]}$. Previous literature reports ${ }^{[7,10]}$, the complications of RFA are mainly associated with needle damage or local thermal damage. While DH after RFA is mainly caused by the injury of the diaphragm by puncture needle, which causes local high temperature in the working state and further aggravates the injury of the diaphragm. After many months, the local diaphragm tension is reduced, and under the attraction of 
continuous negative pressure of the thoracic cavity, the viscera will penetrate into the thoracic cavity through the local weak place of the diaphragm, resulting to $\mathrm{DH}$.

In this case, a DH with acute intestinal obstruction and necrosis occurred 52 months after RFA, after emergency surgery, the patient recovered and was discharged successfully. According to previous literature reports ${ }^{[11-22]}$, the occurrence time of DH after RFA is 7-96 months (median 18 months), combined with our case, DH is considered to be a long-term complication of RFA, which is consistent with the occurrence mechanism of acquired diaphragmatic hernia, suggesting that for patients after RFA, the occurrence of $\mathrm{DH}$ should be vigilant during long-term follow-up.

In some patients with cirrhosis, as the liver atrophy, the space between the liver and the diaphragm gradually becomes larger, and the intestine embedded in it, which is known as Chilaiditi Syndrome. When the diaphragm is locally weak due to RFA, the intestines in this space can herniate into the chest from it, causing diaphragmatic hernia. In this case, the presence of Chilaiditi Syndrome was consistently observed during CT follow-up after RFA, which eventually led to $\mathrm{DH}$. In a review of previous literature ${ }^{[11-}$ 22], we found that Chilaiditi Syndrome was present in most cases of DH after RFA. Therefore, high vigilance should be paid to the occurrence of subsequent DH when Chilaiditi Syndrome was shown on CT during the follow-up of RFA.

A review of the previous 12 cases of $\mathrm{DH}^{[11-22]}$ showed that 2 cases chose conservative treatment, including diuresis and albumin supplementation, while 10 cases chose emergency surgery. Among them, 3 cases underwent bowel resection due to necrosis, and most of them had good prognosis. Only 2 cases died, the cause of death was tumor recurrence and liver failure (Table 1). The patient in this case was in good preoperative condition, with Child-Pugh A, although intestinal necrosis had been complicated, the patient had a good body tolerance and recovered well after surgery. By reviewing the literature, for patients with DH after RFA, when there is no bowel incarcerated or the patient's general condition is poor, the doctors can consider to conservative treatment, although the short-term curative effect, but in the long run, because failing to repair the diaphragm local weak place, under the condition of prolonged negative pressure in the thoracic cavity, $\mathrm{DH}$ is prone to persist and recurring attacks, to avoid the possibility of emergency surgery in the next attack, we recommend that diaphragmatic repair should be performed in time after the general condition of the patient is improved, and laparoscopic surgery can be considered when conditions permitted to reduce injury.

\section{Conclusion}

Finally, we conclude that diaphragmatic hernia $(\mathrm{DH})$ is a long-term complication of RFA. For patients with DH after RFA, when combined with acute completely mechanical obstruction or intestinal necrosis, emergency surgery should be performed. However, when the obstruction is mild and the general condition is poor, conservative treatment can be considered, and diaphragmatic repair should be performed at an appropriate time after the general condition is improved. 


\section{Abbreviations}

\begin{tabular}{|ll|}
\hline RFA & Radiofrequency ablation \\
\hline HCC & Hepatocellular carcinoma \\
\hline TACE & Transcatheter arterial chemoembolization \\
\hline CT & Computed tomography \\
DH & Diaphragmatic hernia \\
AFP & Alpha fetoprotein \\
\hline NCCN & National Comprehensive Cancer Network \\
\hline
\end{tabular}

\section{Declarations}

Ethics approval and consent

This article was approved by the Ethics Committee Review Board of Shaoxing People's Hospital (Ethical No. 2020-K-Y-147-01).

Consent for publication

We had obtained informed consent from the patient before the study began, and consent form was signed by the patient.

Availability of data and materials

All data and materials are available within the manuscript.

Competing interests

The authors declare that they have no conflict of interest to disclose. All authors have read this manuscript and considered for publication. None of the material related to this manuscript has been published or is under consideration for publication elsewhere, including the internet.

Funding

This article was supported by General Research Program of Zhejiang Provincial Department of Health (Grant No. 2021KY1150).

Authors' contributions

LYQ, TQL and XGG performed the operation and managed the patient. LYQ wrote the manuscript. SLYJ consulted the abdominal CT and partly wrote the manuscript. YSL provided the pathological picture. 


\section{References}

1. Bray F, Ferlay J, Soerjomataram I, et al. Global cancer statistics 2018: GLOBOCAN estimates of incidence and mortality worldwide for 36 cancers in 185 countries. CA Cancer J Clin. 2018;68:394424.

2. Kirstein MM, Wirth TC. Multimodal treatment of hepatocellular carcinoma. Internist (Berl). 2020 Feb;61(2):164-9.

3. Lencioni R, Crocetti L. Local-regional treatment of hepatocellular carcinoma. Radiology. 2012 Jan;262(1):43-58.

4. Gupta P, Maralakunte M, Kumar-M P, et al. Overall survival and local recurrence following RFA, MWA, and cryoablation of very early and early HCC: a systematic review and Bayesian network metaanalysis. Eur Radiol. 2021 Jan 13. doi:10.1007/s00330-020-07610-1.

5. Hara K, Takeda A, Tsurugai Y, et al. Radiotherapy for Hepatocellular Carcinoma Results in Comparable Survival to Radiofrequency Ablation: A Propensity Score Analysis. Hepatology. 2019 Jun;69(6):2533-45.

6. Lei JY, Yan LN, Li DJ, et al. Comparison of open liver resection and RFA for the treatment of solitary 3-5-cmhepatocellular carcinoma: a retrospective study. BMC Surg. 2019 Dec 16;19(1):195.

7. Maeda M, Saeki I, Sakaida I, et al. Complications after Radiofrequency Ablation for Hepatocellular Carcinoma: A Multicenter Study Involving 9,411 Japanese Patients. Liver Cancer. 2020 Jan;9(1):5062.

8. Suwa K, Seki T, Tsuda R, et al. Short term treatment results of local ablation with water-cooled microwave antenna for liver cancer: Comparison with radiofrequency ablation. Mol Clin Oncol. 2020 Mar;12(3):230-6.

9. Saraswat VA, Pandey G, Shetty S. Treatment algorithms for managing hepatocellular carcinoma. J Clin Exp Hepatol. 2014 Aug;4(Suppl 3):80-9.

10. lezzi R, Pompili M, Posa A, et al. Interventional oncology treatments for unresectable early stage HCC in patients with a high risk for intraprocedural bleeding: Is a single-step combined therapy safe and feasible? Eur J Radiol. 2019 May;114:32-7.

11. Koda M, Ueki M, Maeda N, et al. Diaphragmatic perforation and hernia after hepatic radiofrequency ablation. AJR Am J Roentgenol. 2003 Jun;180(6):1561-2.

12. Shibuya A, Nakazawa T, Saigenji K, et al. Diaphragmatic hernia after radiofrequency ablation therapy for hepatocellular carcinoma. AJR Am J Roentgenol. 2006 May;186(5 Suppl):241-3.

13. Francesco FD, Sandro SD, Doria C, et al. Diaphragmatic hernia occurring 15 months after percutaneous radiofrequency ablation of a hepatocellular cancer. Am Surg. 2008 Feb;74(2):129-32.

14. Yamagami T, Yoshimatsu R, Matsushima S, et al. Diaphragmatic hernia after radiofrequency ablation for hepatocellular carcinoma. Cardiovasc Intervent Radiol. 2011 Feb;34(Suppl 2):175-7.

15. Singh M, Singh G, Pandey A, et al. Laparoscopic repair of iatrogenic diaphragmatic hernia following radiofrequency ablation for hepatocellular carcinoma. Hepatol Res. 2011 Nov;41(11):1132-6. 
16. Boissier F, Labbé V, Marchetti G, et al. Acute respiratory distress and shock secondary to complicated diaphragmatic hernia. Intensive Care Med. 2011 Apr;37(4):725-6.

17. Kim JS, Kim HS, Myung DS, et al. A case of diaphragmatic hernia induced by radiofrequency ablation for hepatocellular carcinoma. Korean J Gastroenterol. 2013 Sep;62(3):174-8.

18. Zhou MQ, He HF, Cai HK, et al. Diaphragmatic perforation with colonic herniation due to hepatic radiofrequency ablation: A case report and review of the literature. Oncol Lett. 2013 Dec;6(6):171922.

19. Nomura R, Tokumura H, Furihata M. Laparoscopic repair of a diaphragmatic hernia associated with radiofrequency ablation for hepatocellular carcinoma: lessons from a case and the review of the literature. Int Surg Jul-Aug. 2014;99(4):384-90.

20. Nakamura T, Masuda K, Thethi RS, et al. Successful surgical rescue of delayed onset diaphragmatic hernia following radiofrequency ablation for hepatocellular carcinoma. Ulus Travma Acil Cerrahi Derg. 2014 Jul;20(4):295-9.

21. Saito T, Chiba T, Ogasawara S, et al. Fatal Diaphragmatic Hernia following Radiofrequency Ablation for Hepatocellular Carcinoma: A Case Report and Literature Review. Case Rep Oncol. 2015 May 28;8(2):238 - 45 .

22. Abe $\mathrm{T}$, Amano $\mathrm{H}$, Takechi $\mathrm{H}$, et al. Late-onset diaphragmatic hernia after percutaneous radiofrequency ablation of hepatocellular carcinoma: a case study. Surg Case Rep. 2016 Dec;2(1):25.

\section{Tables}

Due to technical limitations, table 1 is only available as a download in the Supplemental Files section.

\section{Figures}

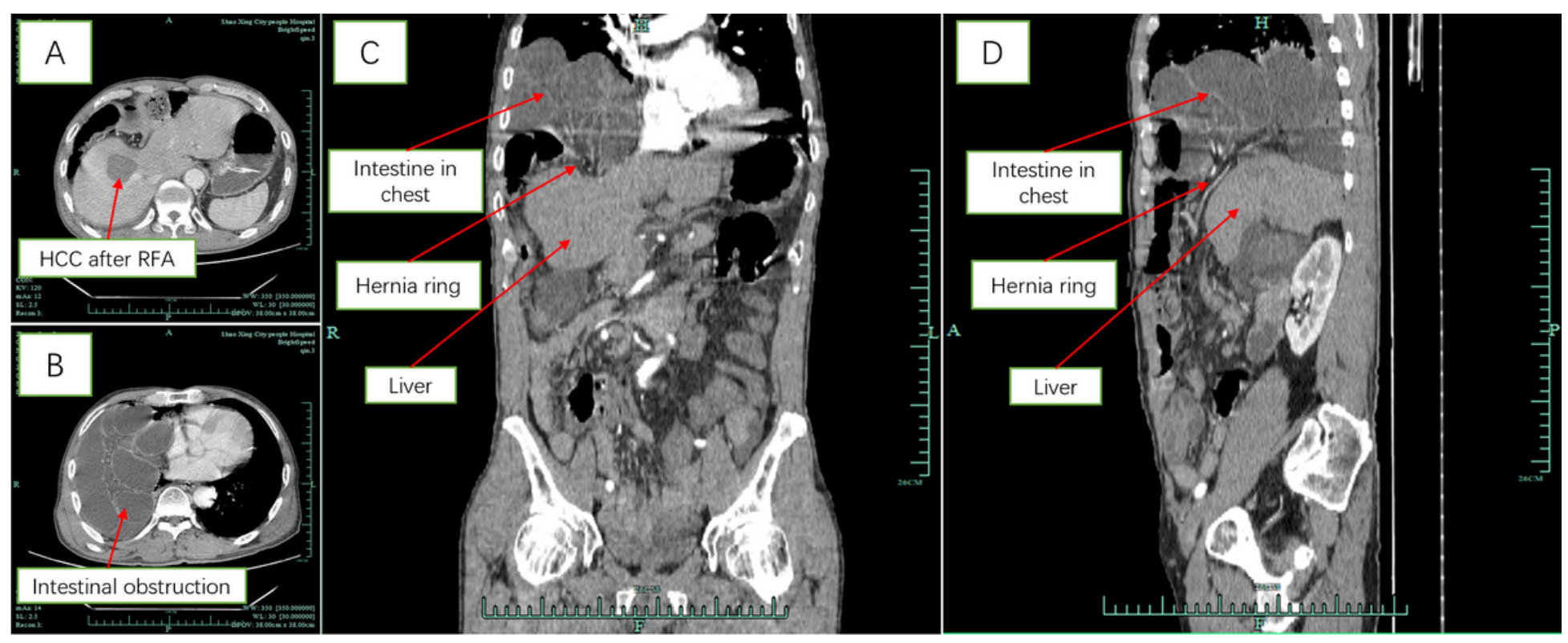

Figure 1 
Abdominal computed tomography (CT). A, HCC in S8 after RFA and TACE. B, the small intestine incarcerated in the chest cavity. C, coronal scan of abdominal CT. D, sagittal scan of abdominal CT.
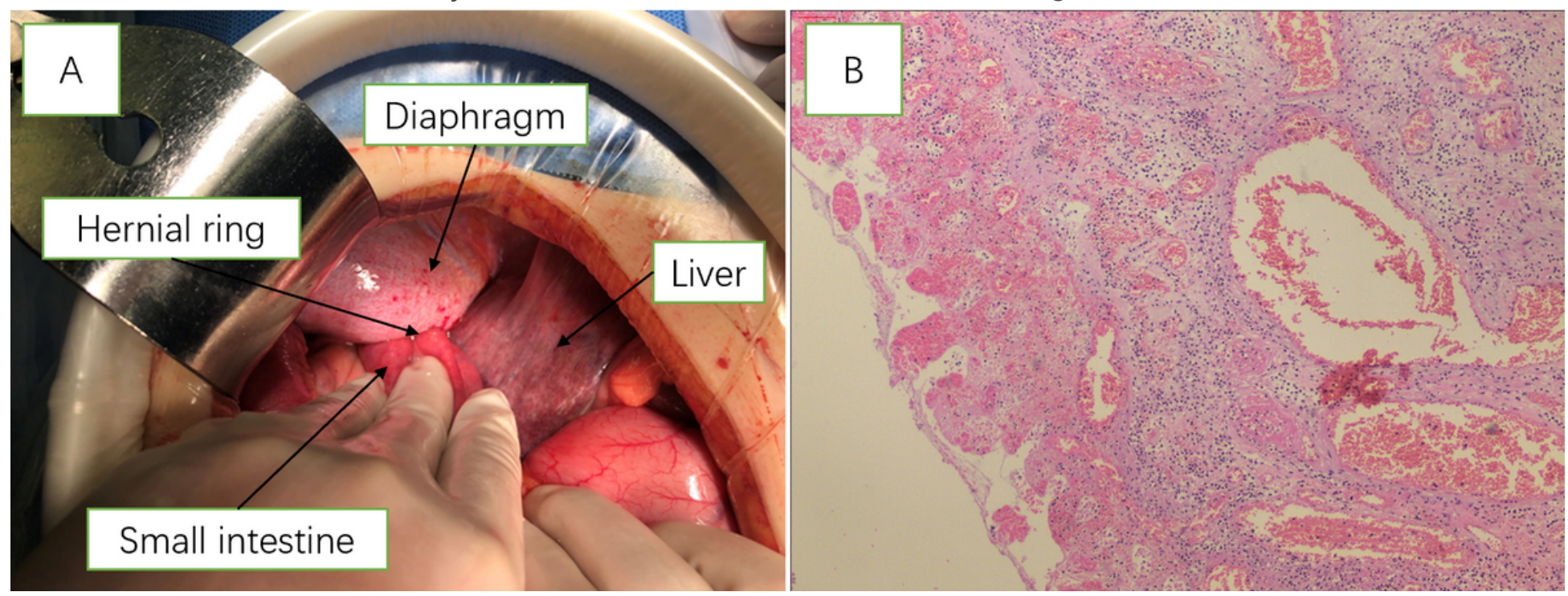

\section{Figure 2}

A, the operative photograph showed a diaphragmatic hernia. B, the postoperative pathologic images showed intestinal necrosis.

\section{Supplementary Files}

This is a list of supplementary files associated with this preprint. Click to download.

- Table1.xlsx 